\title{
Health Behavior Change for Obesity Management
}

\author{
Pedro J. Teixeira ${ }^{a} \quad$ Marta M. Marques ${ }^{a, b}$ \\ ${ }^{a}$ Interdisciplinary Center for the Study of Human Performance (CIPER), Self-Regulation \\ Group, Faculty of Human Kinetics, University of Lisbon, Cruz Quebrada/Dafundo, Portugal; \\ a,b UCL Centre for Behaviour Change University College London, London, UK
}

\section{Keywords}

Behavior modification - Behavioral interventions - Obesity management · Psychological aspects

\begin{abstract}
Health behavior change is central in obesity management. Due to its complexity, there has been a growing body of research on: i) the factors that predict the adoption and maintenance of health behaviors, ii) the development and testing of theories that conceptualize relationships among these factors and with health behaviors, and iii) how these factors can be implemented in effective behavior change interventions, considering characteristics of the content (techniques) and delivery. This short review provides an overview of advances in behavior change science theories and methods, focusing on obesity management, and includes a discussion of the main challenges imposed by this research field.
\end{abstract}

(C) 2017 The Author(s)

Published by S. Karger GmbH, Freiburg

\section{Introduction}

Successfully influencing individual health behaviors has never been as important as it is today, mainly because of the well-known effects of these behaviors in the prevention and management of various health conditions, and due to the increased importance placed on individual autonomy and capacity to self-regulate their own health. Reducing overweight and obesity are key public health challenges. The World Health Organization (WHO) [1] estimates that $39 \%$ of adults worldwide are overweight and $13 \%$ obese, leading to a range of health complications as well as increased health costs. A recent meta-analysis led by our research laboratory [2] examining the prevalence of weight control attempts worldwide (72 studies; 
$\mathrm{n}=1,189,942$ ) showed that $42 \%$ of adults from general population and $44 \%$ from ethnicminority populations are trying to lose weight, and 23\% reported trying to maintain their weight at some point. Behavioral interventions targeting changes in diet and physical activity are the cornerstone of interventions for weight management in overweight and obese populations [3] and seem to be effective in reducing weight and improving health at least in the short term (e.g. [4]).

The emergence and rapid growth of the health behavior change field is one response to the urgent need to understand the complexity behind individuals' decisions and engagement in behaviors that affect their health and well-being, including sustained weight management. Health behavior change interventions (HBCIs) have the potential to improve the health of populations if they can be scaled up and appropriately targeted, considering issues like difficulty and motivation for change [5]. Since interventions are meant for the real world, context sensitivity is paramount. In other words, an intervention is only as successful as its capacity to adequately respond to a problem in an environment for a certain target population and focused on certain behavioral outcome(s). Evidence-based practice health behavior change therefore depends on the adequate development and implementation of interventions [6], making use of standardized methods to report them [7].

In this short narrative review, we will present some of the most current topics of research in the field of health behavior change, with a focus on the management of obesity, including i) the use of formal theories and a correct consideration of their mechanisms of action, ii) the choice of the behavior change techniques (or 'active ingredients') included in HBCIs, and iii) the use of technology to promote sustained behavior change.

\section{The Role of Theory and Mechanisms of Action}

Theories ('systematic way of understanding events or situations, (...) a set of concepts, definitions, and propositions that explain or predict these events or situations by illustrating the relationships between variables' [8], p. 4) are useful to understand, explain, and predict behavior and behavior change, as they conceptualize a set of interrelated constructs operating as predictors or mechanisms of action underlying behavior change. There are various levels of constructs that influence health behavior; they are therefore conceptualized in health behavior change theories. These can be done at the environmental level whether it is physical, cultural, or social (e.g., advice from a healthcare practitioner, low accessibility, peer support) or at the individual level including biological factors (e.g., food reward mechanisms) but also emotions, motivation, and self-regulation skills. Individual factors are considered fundamental for health behavior change as they are mostly responsible for the process of selfregulation of health behaviors. For instance, a systematic review looking at psychological mediators of sustained beneficial effects in lifestyle obesity interventions [9] found that higher levels of autonomous motivation, self-efficacy/barriers, self-regulation skills, flexible eating restraint, and positive body image were mediators of medium-/long-term weight control. High autonomous motivation, self-efficacy, and use of self-regulation skills were significant mediators of physical activity while for dietary intake no consistent mediators were identified.

Recently, a broad consensus emerged indicating that HBCIs can be optimized if they are informed by theory [10], as it facilitates the understanding of what works to change a certain behavior and how it works [11]. Theories of behavior change propose the mechanisms of action (under the broad categories of capacity, opportunity, and motivation) and the moderators of change through causal predictions. While there is an agreement in health behavior change that the use of theory is useful to promote long-lasting behavior change, there is still 
limited research on the effectiveness of theory-based (vs. non-theory-based) interventions. For instance, a recent meta-analysis by Gourlan and colleagues [12] investigated the effects of 82 theory-based randomized controlled trials targeting physical activity and showed beneficial but small effects of theory-based interventions in changing physical activity $(d=0.31)$. Similar results were found in a meta-analysis of digital-based interventions targeting various health behaviors (85 studies), in which the extensive use of theory (e.g., use theoretical constructs to develop intervention techniques) was associated with larger intervention effects [13]. Another meta-analysis examining the influence of theory use in physical activity and dietary interventions, did not find significant associations (e.g. [14]). There are several reasons that may explain these results: i) limited number of theories commonly tested (e.g., Social Cognitive Theory, Theory of Planned Behavior), ii) the fact that some theories may not provide a clear explanation on the process of behavior change maintenance, and iii) when interventions are explicitly based on theory, they often do not apply it extensively $[12,14]$. Furthermore, research findings suggest that single-theory approaches may be more effective in influencing behaviors such as physical activity, comparing with those interventions applying multiple theories $[12,13]$. This finding may be related to the fact that some interventions consist of a combination of two or more theories (or key constructs from these theories) lacking internal coherence and parsimony [15].

One of the problems faced when intending to use theory in HBCIs is the large number of theories that currently exist. Recently, a panel of experts has identified and compiled 83 formal theories of behavior and behavior change (including more than 1,700 theoretical constructs) in a comprehensive compendium [16]. Faced with so many theories from which to select from, researchers and practitioners need the skills to make decisions regarding the best candidate theory for a given behavior and context. This can be particularly difficult when targeting multiple behaviors (e.g., physical activity and diet), which is the case when considering weight management interventions. To guide this process, efforts have been made to make frameworks for the development of HBCIs informed by theory. This includes the Intervention Mapping Protocol [17] or the Theoretical Domains Framework [18]. In addition, tools such as the Theory Coding Scheme allow for an evaluation of the extension of use of theory in a HBCI [19]. The overarching COM-B model [6], which contains three broad theory-related dimensions of behavior change determinants - competence, motivation, and opportunity -, can also be used to make decisions on the design of HBCIs, especially when this is conducted without input by health psychologists or behavior change specialists.

While behavioral interventions seem to be effective in promoting weight loss, weight loss maintenance is a key challenge as most adults that successfully lose weight tend to regain part of it within 1 year [20]. Currently, there are very few comprehensive treatments available, and indeed most of the research has focused on the behavioral aspects associated with weight loss $[21,22]$. A recent systematic review on theoretical explanations for behavior change maintenance [22] identified five interconnected theoretical explanations about how individuals maintain initial behavior changes over time: i) maintenance motives - tendency to maintain behavior when there are sustained motives (e.g. enjoyment) and congruence between behavior and identity/values (e.g. self-determination theory [23]); ii) self-regulation - includes self-monitoring and coping strategies (self-regulation theory [24]); iii) physical and psychological resources (e.g. self-control theory [25]), iv) habit - habitual behaviors supported by automatic responses to cues (e.g. habit theory [26]); and v) environmental and social cues - supportive environment, social support, behavior in line with social changes (e.g. normalization process theory [27]). At present, in long-term weight management there is some support for the effectiveness of HBCIs which are based on self-determination theory (e.g. $[9,28,29])$ and self-regulation theories (e.g. [9, 30, 31]). 


\section{The Active Ingredients of Interventions: Behavior Change Techniques}

A key aspect in the development, implementation and evaluation of HBCIs is the adequate characterization of its content - the 'active ingredients', i.e., the techniques used in interventions to help change another's or one's own behavior. These techniques represent the lowestlevel, irreducible, fundamental elements of an intervention aimed to influence on behavior and are commonly designated behavior change techniques (BCTs) [32]. Some examples of BCTs are 'prompt self-monitoring', 'provide feedback on progress', or 'restructure the environment'. Naturally, complex HBCIs typically involve several of such techniques in various combinations, and detailed taxonomies of BCTs that can be used in HBCIs can be of use in both research and practice, as they promote a shared language between health behavior change researchers and practitioners. Interventions can be described in clearer and more consistent ways and more rigorously tested and compared in research studies, when techniques are reliably used. In turn, practitioners can more easily and consistently be trained in, and be evaluated based on, the use of standardized techniques.

The work led by Michie and colleagues [33,34] is perhaps the most comprehensive and resulted in BCT taxonomies for a range of behaviors, including physical activity, diet, and smoking. More recently, these were collapsed into one overarching list - the BCT Taxonomy v1 - including 93 techniques, organized into 16 higher-level domains [35]. Since the publication of the first BCT taxonomy [36], several meta-analyses of randomized controlled trials have examined the use of BCTs i) looking at the association between the number of BCTs used and the magnitude of the effects, ii) determining which BCTs effectively target certain theoretical constructs, and iii) investigating if certain clusters of theoretically driven BCTs are associated with better results in several health behaviors (e.g. [37]) and health conditions (e.g. $[30,38])$. One of the main reasons for conducting these analyses is that there are typically considerable levels of heterogeneity in the effects of HBCIs. By examining the techniques used in these interventions (as well as the theoretical frameworks that support them), we can select BCTs or clusters of BCTs that can have a higher impact on a certain target behavior under certain conditions, and exclude others in order to develop more effective HBCIs.

Results from reviews suggest that combined use of BCTs can be associated with greater effectiveness. Michie and colleagues [37] found that interventions combining self-monitoring with other BCTs derived from self-regulation theories (e.g. [25]), such as goal setting, provision of feedback, planning and goal revisiting, were more effective in promoting changes in physical activity and healthy eating in the general population than other interventions not using these techniques. Similar effects were found in other meta-analyses, including weight loss and maintenance interventions in overweight/obese subjects (e.g. [30]). In the context of digital-based interventions for weight management, Hutchesson et al. [39] point to the potential beneficial effects of self-monitoring and personalized feedback, and Sherrington and colleagues [40] found that internet-delivered weight loss interventions providing personalized feedback resulted in greater weight loss but only in the short term.

While the BCTT V1 was developed without the consideration of the role of theory in informing the selection and use of BCTs, another common framework for the development of health behavior change interventions - intervention mapping -, clearly states that the selection of techniques should take into consideration the theoretical parameters for its effectiveness [17]. In this respect, taxonomies can be sought for specific theories, where techniques that target the most important constructs of that framework are described. As an example, Teixeira and colleagues [41] are currently developing a comprehensive list of techniques used to influence key self-determination theory constructs.

A better linkage between BCTs and health behavior change theories is a potential benefit since psychological constructs presented in theories are presumably well-targeted by some 
techniques but not (or less so) by others. Techniques are useful in HBCIs to the extent that they impact on the putative mechanisms of action (e.g. goal setting) to change a given behavior (e.g. physical activity). Currently, there are efforts begin made in linking clusters of BCTs to specific mechanisms of action and overarching behavioral theories [42].

\section{Delivery of Health Behavior Change Interventions: Digital Progress}

Another important dimension on the development of effective HBCIs is the delivery of the intervention, which can have an impact on the effectiveness of interventions (e.g. face to face vs. printed material; delivered by psychologist vs. nurse) as well as on the operationalization of certain theories [43]. While there has been a significant progress in specifying BCTs and the mechanisms of action and theoretical frameworks involved in health behavior change, less attention has been given to the elements of delivery. Dombrowski and colleagues [43] propose that 'form of delivery' includes 'all features through which behavior change intervention content is conveyed including: the provider, format, materials, setting, intensity, tailoring and style'. Any HBCI can use a combination of forms and modes of delivery (MoDs). Carey and colleagues [44], define MoD as the way in which BCTs are delivered. They are currently developing a hierarchical classification system in order to specify the MoDs applied in HBCIs, using a similar approach to the development of the BCTT V1. For example, the MoD 'informational' includes human, printed material, digital and environmental change; and 'digital' includes technology for delivery (e.g. mobile device) and digital content type (e.g email).

In recent years, there has been a marked increase in the use of digital MoDs in lifestyle interventions for weight management. These are a viable option as they have the potential for wide reach at low cost, which is especially relevant if considered in a large scale and if intended to influence behaviors in the long term (which the case of weight management). Other advantages of using a digital approach are the potential to adapt content to individual needs (personalization), the delivery of information in an engaging and interactive form, and higher degree of fidelity to intervention content $[45,46]$.

While digitally based HBCIs are promising, research on their effects is still in an early stage. In the context of weight management in overweight/obese populations, previous reviews have reported positive albeit often small effects with considerable between-study variability $[13,39,47]$. There is therefore the need to identify which intervention components contribute to the effectiveness of digital-based interventions in promoting sustained weight management $[13,39,40]$. In a meta-analysis of internet-based interventions for health behavior change looking at the characteristics of most effective interventions, theory-based interventions incorporating a larger number of BCTs (vs. interventions with fewer BCTs) and using a variety of MoDs (e.g. internet, SMS) had larger effects on health-related behaviors [13]. The only published meta-analysis looking at the interactions between BCTs and MoDs in digital interventions did not find significant effects [48]. Research focusing on the development of strategies for sustained engagement alongside with health behavior change theory is also a priority for digital interventions [49].

Research on the effectiveness of using digital MoDs in promoting weight loss maintenance is very limited. There are currently two ongoing projects aiming to fill this gap. The first is the 'NoHoW - Evidence-Based ICT Tools for Weight Maintenance' (www.nohow.eu), a European Commission-funded project (Horizon 2020). Following available guidance for the development of complex interventions (e.g. [46, 50]), we developed a toolkit, using evidencebased intervention techniques derived from promising theoretical frameworks in weight loss maintenance, such as self-determination theory, self-regulation theory, and emotion regu- 
lation approaches. The toolkit is currently being tested in the context of a full-factorial randomized controlled trial. It will help us understand if digital-based interventions are an effective way to apply theory and techniques aiming at promoting weight loss maintenance, and which content is more effective for each behavior, for whom, under which circumstances and for which outcomes (Trial Registration: ISRCTN88405328).

The other is the NULevel trial [21], a self-regulatory intervention using automated remote weight-monitoring and feedback system using participants' mobile phones as the main MoD of theory-based BCTs (e.g., self-monitoring, goal setting, coping plans, and increase motivation), and an initial face-to-face behavioral component. NULevel evaluation is currently ongoing.

\section{Conclusion}

There is a scientifically rigorous body of research aiming to identify and improve our understanding of how to effectively develop, implement and evaluate HBCIs, namely in the field of weight management. Researchers have considered effective ways of 'speaking the same language' and to make knowledge accessible for interventionist by developing various taxonomies and frameworks. While considerable progress is evident in this area, there are still many questions to be answered and challenges ahead, as shown for example by the variability of the effects of HBCIs and limited results from meta-analyses examining interactions between intervention features. The Human Behavior-Change Project led by Michie and colleagues (humanbehaviorchange.org) is an example of the most recent efforts in the field of Health behavior change science to promote evidence-based practice [51]. The project consists of a multidisciplinary team of behavioral scientists, computer scientists, and system architects, aiming to build an ontology of behavior change interventions that will classify and organize HBCI features (e.g. BCTs, mechanisms of action, delivery, context) and develop a 'knowledge system' that, through artificial intelligence and machine learning, will automatically extract, synthetize, and interpret information from HBCI research reports, therefore contributing to the design of effective evidence-based interventions [51]. Another landmark project is the US Science of Behavior Change project (scienceofbehaviorchange.org), which also seeks to standardize and synthetize assessment methods and research protocols in the area of human behavior change. It should be noted that classification systems of features of health (and other) behaviors are still a work in progress, and there is ongoing debate on its limitations to capture the complexity of health behavior change [52].

\section{Disclosure Statement}

The authors have no conflicts of interest to declare.

\section{References}

1 World Health Organization: Fact Sheet Obesity and Overweight. 2017. www.who.int/mediacentre/factsheets/ fs311/en/ (last accessed November 29, 2017).

2 Santos I, Sniehotta FF, Marques M, Carraça EV, Teixeira PJ: Prevalence of personal weight control attempts in adults: a systematic review and meta-analysis. Obes Rev 2016;18:32-50. D

3 Jensen MD, Ryan DH, Apovian CM, Ard JD, et al: AHA/ACC/TOS guideline for the management of overweight and obesity in adults: a report of the American College of Cardiology/American Heart Association Task Force on Practice Guidelines and The Obesity Society. Circulation 2014;129(25 suppl 2):S102-138. Erratum in Circulation 2014;129(25 suppl 2):S139-140. 
4 Dombrowski SU, Avenell A, Sniehotta FF: Behavioural interventions for obese adults with additional risk factors for morbidity: systematic review of effects on behaviour, weight and disease risk factors. Obes Facts 2010;3:377-396.

5 Resnicow K, Teixeira PJ, Williams GC: Efficient allocation of public health and behavior change resources: the 'difficulty by motivation' matrix. Am J Publ Health 2017;107:55-57.

6 Michie S, van Stralen M, West R: The behaviour change wheel: a new method for characterising and designing behaviour change interventions. Implement Sci 2011;6:42.

7 Hoffmann T, Glasziou P, Boutron I, Milne R, et al: Better reporting of interventions: template for intervention description and replication (TIDieR) checklist and guide. BMJ 2014;348:1687.

8 Glanz K, Rimer BK: Theory at a Glance: A Guide for Health Promotion Practice, 2nd ed. Washington DC, U.S. Government Printing Office, 2005.

9 Teixeira PJ, Carraça EV, Marques MM, Rutter H, et al: Successful behavior change in obesity interventions in adults: A systematic review of self-regulation mediators. BMC Med 2015;13:84.

10 Michie S, Johnston M, Abraham C, Lawton R, et al: Making psychological theory useful for implementing evidence based practice: a consensus approach. Qual Saf Health Care 2005;14:26-33.

11 Michie S, Abraham C: Interventions to change health behaviours: evidence-based or evidence-inspired? Psychol Health 2004;19:29-49.

12 Gourlan M, Bernard P, Bortholon C, Romain AJ, et al: Efficacy of theory-based interventions to promote physical activity. A meta-analysis of randomised controlled trials. Health Psychol Rev 2015;10:1-17.

13 Webb T, Joseph J, Yardley L, Michie S: Using the internet to promote health behavior change: a systematic review and meta-analysis of the impact of theoretical basis, use of behavior change techniques, and mode of delivery on efficacy. J Med Int Res 2010;12:e4.

14 Prestwich A, Sniehotta F, Whittington C, Dombrowski S: Does theory influence the effectiveness of health behavior interventions? Meta-analysis. Health Psychol 2014;33:465-474.

15 Silva MN, Marques MM, Teixeira PJ: Testing theory in practice: the example of self-determination theorybased interventions. Euro Health Psychol 2014;16:171-180.

16 Michie S, West R, Campbell R, Brown J, Gainforth H: ABC of Behaviour Change Theories: An Essential Resource for Researchers, Policy Makers and Practitioners. Bream, Silverback Publishing, 2014.

17 Kok G, Gottlieb NH, Peters G-J Y, Mullan PD, et al: A taxonomy of behaviour change methods: an intervention mapping approach. Health Psychol Rev 2016;10:297-312.

18 Cane J, O'Connor D, Michie S: Validation of the theoretical domains framework for use in behaviour change and implementation research. Implement Sci 2012;7:37.

19 Michie S, Prestwich A: Are interventions theory-based? Development of a theory coding scheme. Heal Psychol 2010;29:1-8.

20 Avenell A, Broom J, Brown TJ, Poobalan A, Aucott L, Stearns SC, et al: Systematic review of the long-term effects and economic consequences of treatments for obesity and implications for health improvement. Health Technol Assess 2004;8:1-182.

21 Evans E, Araujo-Soares V, Adamson A, Batterham AM, Brown H, Campbell M, et al: The NULevel trial of a scalable, technology-assisted weight loss maintenance intervention for obese adults after clinically significant weight loss: study protocol for a randomised controlled trial. Trials 2015;16:421.

22 Kwasnicka D, Dombrowski SU, White M, Sniehotta S: Theoretical explanations for maintenance of behaviour change: a systematic review of behaviour theories, Health Psychol Rev 2015;34:1191-1194.

23 Deci E, Ryan R: The 'what' and 'why' of goal pursuits: human needs and the self- determination of behavior. Psychol Inquir 2000;11:227-268.

24 Carver CS, Scheier MF: Control theory: a useful conceptual framework for personality-social, clinical, and health psychology. Psychol Bull 1982;92:111-135.

25 Muraven M, Baumeister RF: Self-Regulation and depletion of limited resources: does self-control resemble a muscle? Psychol Bull 2000;126:247-259.

26 Verplanken B, Aarts H: Habit, attitude, and planned behaviour: is habit an empty construct or an interesting case of goal-directed automaticity? Eur Rev Soc Psychol 1999;10:101-134.

27 May CR, Mair F, Finch T, MacFarlane A, et al: Development of a theory of implementation and integration: Normalization Process Theory. Implem Sci 2009;4:29.

$28 \mathrm{Ng} J Y$, Ntoumanis N, Thøgersen-Ntoumani C, Deci E, et al: Self-determination theory applied to health contexts: a meta-analysis. Perspect Psychol Sci 2012;7:325-340.

29 Teixeira PJ, Carraça EV, Markland DM, Silva MN, Ryan R: Exercise, physical activity, and self-determination theory: a systematic review. Int J Behav Nutri Phys Act 2012;9:78.

30 Dombrowski SU, Sniehotta F, Avenell A, Johnston M, MacLennan G, Araujo-Soares V: Identifying active ingredients in complex behavioural interventions for obese adults with obesity-related co-morbidities or risk factors for co-morbidities: a systematic review. Health Psych Rev 2012;6:7-32.

31 Wing RR, Tate DF, Gorin AA, Raynor HA, Fava JL: A self-regulation program for maintenance of weight loss. N Engl J Med. 2006;355:1563-1571.

32 Michie S, Abraham C, Eccles MP, Francis JJ, Hardeman W, Johnston M: Strengthening evaluation and implementation by specifying components of behaviour change interventions: a study protocol. Implem Sci 2011; $6: 10$. 
33 Michie S, Ashford S, Sniehotta FF, Dombrowski SU, Bishop A, French DP: A refined taxonomy of behaviour change techniques to help people change their physical activity and healthy eating behaviours: the CALO-RE taxonomy. Psychol Health 2011;26:1479-1498.

34 Michie S, Hyder N, Walia A, West R: Development of a taxonomy of behaviour change techniques used in individual behavioural support for smoking cessation. Addict Behav 2011;36:315-319.

35 Michie S, Richardson M, Johnston M, Abraham C, et al: The Behavior Change Technique Taxonomy (v1) of 93 hierarchically clustered techniques: building an international consensus for the reporting of behavior change interventions. Ann Behav Med 2013;46:81-95.

36 Abraham C, Michie S: A taxonomy of behavior change techniques used in interventions. Health Psych 2008; 27:379-387.

37 Michie S, Abraham C, Whittington C, McAteer J, Gupta S: Effective techniques in healthy eating and physical activity interventions: a meta-regression. Health Psychol 2009;28:690-701.

38 Jansen, V, de Gucht, V, Dusseldorp E, Maes S: Lifestyle modification programmes for patients with coronary heart disease: a systematic review and meta-analysis of randomized controlled trials. Eur J Prev Cardiol 2013; 20:620-640.

39 Hutchesson MJ, Rollo ME, Krukowski R, Ellis L, et al: eHealth interventions for the prevention and treatment of overweight and obesity in adults: a systematic review with meta-analysis. Obes Rev 2015;16:376-392.

40 Sherrington A, Newman JJ, Bell R, Adamson A, McColl E, Araujo-Soares V: Systematic review and meta-analysis of internet delivered interventions providing personalized feedback for weight loss in overweight and obese adults. Obes Rev 2016;17:541-551.

41 Teixeira PJ, Hagger M: Motivation and behaviour change techniques based on self-determination theory: a consensus analysis. Eur Health Psychol 2016;18(suppl):389.

42 Michie S, Carey R, Johnston M, Rothman A, de Bruin M, Kelly, M, Connell L: From theory-inspired to theorybased interventions: a protocol for developing and testing a methodology for linking behaviour change techniques to theoretical mechanisms of action. Annals Behav Med 2016; doi: 10.1007/s12160-016-9816-6.

43 Dombrowski SU, O'Carroll, RE, Williams B: Form of delivery as a key 'active ingredient' in behaviour change interventions. Br J Health Psychol 2016;21:733-740.

44 Carey RN, Evans F, Horan M, Johnston M, West R, Michie S: Describing the 'how' of behaviour change interventions: a taxonomy of modes of delivery. Paper presented at the UK Society for Behavioural Medicine Conference, Cardiff, UK, 2016. www.kc-jones.co.uk/files/uploads/1481819318.pdf (last accessed November 29, 2017).

45 Griffits F, Lindenmeyer A, Powell J, Lowe P, Thorogood M: Why are health care interventions delivered over the internet? A systematic review of the published literature. J Med Intern Res 2016;8:e1.

46 Michie S, West R: A Guide to Development and Evaluation of Digital Behaviour Change Interventions in Healthcare. UCL Centre for Behaviour Change Monograph. Bream, Silverback Publishing, 2016.

47 Neve M, Morgan PJ, Jones RE, Collins CE: Effectiveness of web-based interventions in achieving weight loss and weight loss maintenance in overweight and obese adults. Obes Rev 2010;11:306-321.

48 van Genugten L, Dusseldorp E, Webb T, van Empelen P: Which combinations of techniques and modes of delivery in internet-based interventions effectively change health behavior? A meta-analysis. J Med Intern Res 2016;18:e155.

49 Kelders SM, Kok RN, Ossebaard HC, Van Gemert-Pijnen J: Persuasive system design does matter: a systematic review of adherence to web-based interventions. J Med Intern Res; 2012;14: e152.

50 Craig P, Dieppe P, Macintyre S, Michie S, Nazareth I, Petticrew M: Developing and evaluating complex interventions: the new Medical Research Council guidance. BMJ 2008;337:a1655

51 Michie S, Thomas J, Johnston M, Aonghusa PM, Shawe-Taylor J, Kelly MP, Deleris LA, Finnerty MM, Norris E, O'Mara-Eves A, West R: The Human Behaviour-Change Project: Harnessing the power of artificial intelligence and machine learning for evidence synthesis and interpretation. Implem Sci 2017;12:121.

52 Ogden J: Celebrating variability and a call to limit systematisation: the example of the behaviour change technique taxonomy and the behaviour change wheel. Health Psych Rev 2016;10:245-250. 